\title{
In-Situ Observation of the Reversible Electrochemical Deposition of Fe in a Transmission Electron Microscope
}

\author{
U. Wolff ${ }^{1}$, B. Ambrožič ${ }^{2}$ K. Žužek Rožman ${ }^{2}$, K. Leistner ${ }^{1}$, K. Nielsch ${ }^{1}$ and S. Šturm ${ }^{2}$ \\ 1. Leibniz Institute for Solid State and Materials Research, Institute for Metallic Materials, Dresden, \\ Germany \\ 2. Jožef Stefan Institute, Department for Nanostructured Materials, Ljubljana, Slovenia
}

Recently, materials science benefits largely from in-situ transmission electron microscopy (TEM) for the investigation of atomistic reaction mechanisms at interfaces. For in-situ TEM, specialized holders have been developed, which separate the sample and surrounding liquids or gases from the high vacuum of the microscopy column. With these novel approaches, the mechanism of dynamical processes can be revealed, reaction kinetics can be quantified, and even electrochemical processes can be studied in-situ in the microscope [1,2]. So far, only the in-situ electrodeposition of noble metals such as $\mathrm{Pt}$ and $\mathrm{Cu}$ are investigated [3]. We are interested in the electrodeposition of $\mathrm{Fe}$, which has regained increasing attention because of the unique catalytic, electronic, and magnetic properties of Fe nanostructures. Tailored shapes of Fe nanoparticles and self-termination phenomena in ultrathin films are currently discussed with regard to the role of hydrogen evolution that accompanies Fe electrodeposition [4,5]. The detailed atomistic processes involved are still under debate. Therefore, we investigate the initial stages of Fe electrodeposition by in-situ TEM. A glassy carbon electrode has been used as a working electrode and Pt as reference and counter electrode in a liquid TEM cell. The experiments were performed on a JEM-2100 (JEOL Ltd., Tokyo, Japan) microscope operated at $200 \mathrm{kV}$, which is equipped with an energy dispersive X-ray spectrometer (EDXS). A Poseidon 510 TEM holder (Protochips Inc., Raleigh, NC, USA) was used with a Gamry Reference 600 potentiostat (Gamry Instruments, Warminster, PA, USA) for conducting the electrochemical experiments (chronoamperometry). The liquid cell consists of two chips with $50 \mathrm{~nm}$ thin amorphous $\mathrm{SiN}_{\mathrm{x}}$ windows, where the smaller one has a spacer height of $50 \mathrm{~nm}$, thus controlling the total thickness of the liquid layer between the two windows. The upper, larger chip contains the glassy carbon working electrode, the $\mathrm{Pt}$ reference and the $\mathrm{Pt}$ counter electrode. A Fe sulphate aqueous solution with addition of sodium citrate and sodium sulphate at a $\mathrm{pH}=2$ was used as electrolyte. Applying a potential of $-1.1 \mathrm{~V}$ vs. Pt stimulates the electrodeposition of amorphous islands of Fe preferably at the edges of the glassy carbon working electrode, which subsequently crystallize to $\mathrm{Fe}$ and $\mathrm{FeO}_{\mathrm{x}} / \mathrm{Fe}(\mathrm{OH})_{\mathrm{x}}$, respectively. The nucleation of the crystal growth occurs instantaneously upon reaching a critical potential. The resulting deposition is discontinuous. Switching the potential to $-0.8 \mathrm{~V}$ vs. Pt leads to the dissolution of the previously formed species. The deposition-dissolution process is repeatable for many cycles (Fig. 1). Radial integration of the electron diffraction patterns reveals the presence of crystalline $\gamma$-Fe (fcc), which was confirmed by fitting the experimental selected area diffraction pattern (SAED, Fig. 2). In order to minimize artifacts such as the beam-induced radiolysis of water or secondary radical chemistry, the electron beam was blocked during the electrodeposition. We have successfully shown for the first time the in-situ Fe deposition-dissolution process in a liquid TEM cell and affirmed that the Fe particles grow through electrochemical deposition rather than by virtue of the electron beam. The nucleation, morphology, and composition of the deposited Fe particles are discussed [6]. 


\section{References:}

[1] BL Mehdi et al, Nano Letters 15 (2015), p. 2168.

[2] L Wang et al, Nanoscale 8 (2016), p. 17250.

[3] GZ Zhu et al, J. Phys. Chem. C 118 (2014), p. 22111.

[4] K Leistner et al, Nanoscale 9 (2017), p. 5315.

[5] R Wang et al, J. Phys. Chem. C 120 (2016), p. 16228.

[6] Dr. B Rellinghaus is thanked for many fruitful discussions to this work.

\section{$\mathrm{E}=-1.1 \mathrm{~V} / \mathrm{Pt}$ for $1500 \mathrm{~s}$}

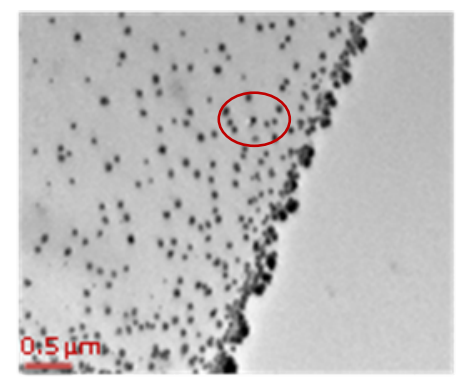

$1200 \mathrm{~s}$

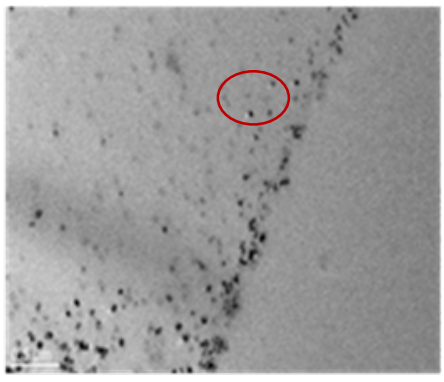

$\mathrm{E}=-0.8 \mathrm{~V} / \mathrm{Pt}$ for $300 \mathrm{~s}$

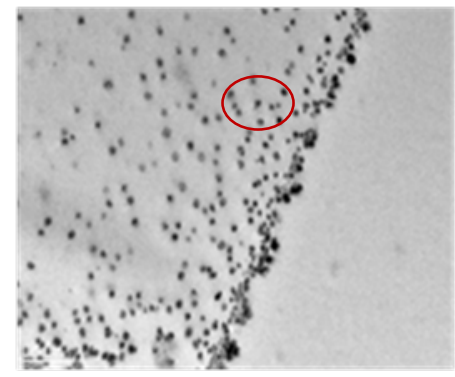

$\mathrm{E}=-1.1 \mathrm{~V} / \mathrm{Pt}$ for $600 \mathrm{~s}$

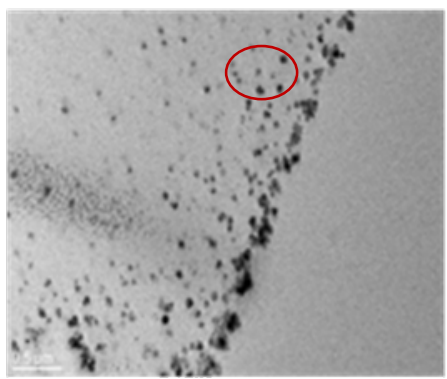

$900 \mathrm{~s}$

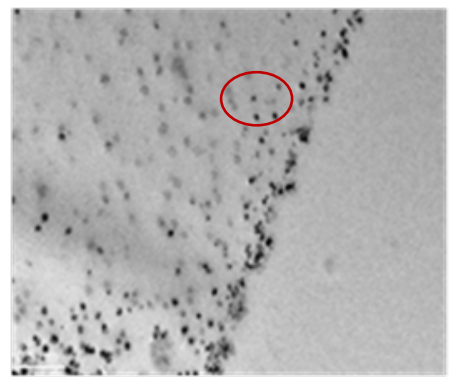

$\mathrm{E}=-0.8 \mathrm{~V} / \mathrm{Pt}$ for $1800 \mathrm{~s}$

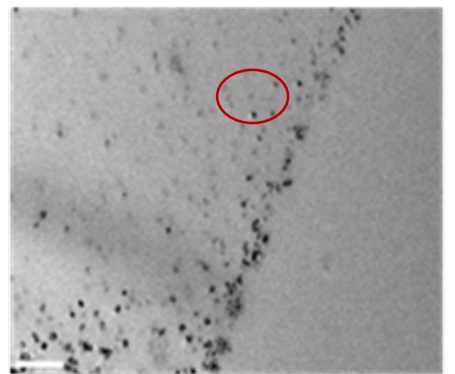

Figure 1. Series of TEM images showing the reversible deposition $(E=-1.1 \mathrm{~V})$ and dissolution $(E=-0.8$ $\mathrm{V})$ of Fe particles

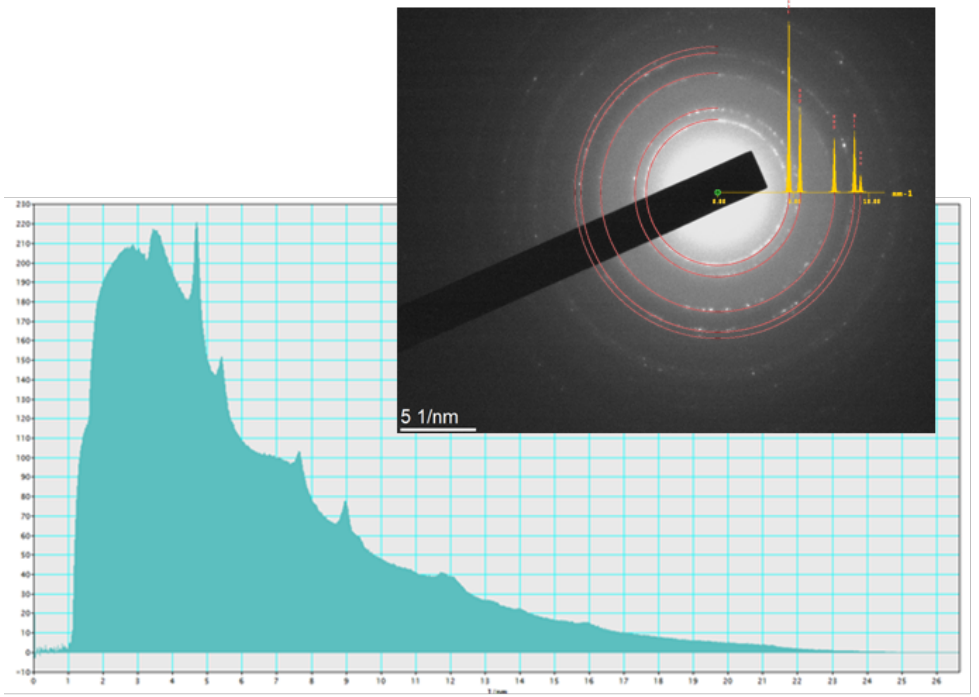

\begin{tabular}{|c|c|}
\hline $\begin{array}{c}\text { exp. data } \\
\text { SAED }\end{array}$ & $\gamma$-Fe 44862 \\
\hline $\mathrm{nm}-1$ & $\mathrm{~nm}-1$ \\
\hline 4.66 & 4.75 \\
\hline 5.37 & 5.48 \\
\hline 7.62 & 7.75 \\
\hline 8.96 & 9.09 \\
\hline 11.8 & 11.95 \\
\hline 14.06 & 14.0 \\
\hline 16.2 & 16.05 \\
\hline
\end{tabular}

Figure 2. Experimental and fitted/integrated SAED pattern revealing the existence of crystalline $\gamma$-Fe 